\title{
reviews
}

\section{Immunological sourcebook}

Lymphocyte Differentiation, Recognition and Regulation. By David $\mathrm{H}$. Katz. Pp. xiii + 749. (Academic: London and New York, 1977.) \$42; £29.80.

THE scope of this book is no less than a review of the whole of current basic immunology. This is approached under a series of chapter headings dealing with the surface antigens of $T$ and $B$ cells and their respective ontogenies; receptors and specificity of these two major subclasses and their functional properties; regulatory interactions in immune responses, including genetic control and the role of the major histocompatibility complex; and finally, immunological tolerance.

This is a monumental assignment for one person to undertake. Two thousand three hundred references are quoted, most of them published since 1970. Dr Katz's approach has been to consider his selection of the relevant references under the headings he has chosen, in many cases quoting substantial details of the work under discussion. In chapters where he has integrated the results of several papers and the views of different authors, the story emerges as an interesting one, and his own views are illuminating. This successful approach is seen most in the chapters dealing with T-B cell cooperation, a field in which the author has been extensively engaged. One can argue with him as to his choice of relevant references, and the reservations, many times reiterated, with which he regards data gathered from in vitro systems, but the overall synthesis of these chapters is good; they are both informative and provocative.

The chapters dealing with subjects outside the author's own experimental field suffer from repetition and a lack of synthesis of the data and in the discussion presented. Some sections read like a catalogue of papers; for example, the section on $\mathrm{H}$-2-restricted cytotoxic responses describes sequentially and in great detail each of the examples (this class of response to virus, hapten and minor transplantation antigens), and fails to underline the essential similarity of the systems and the important biological implications of the findings. The general lack of cross-referencing, either in the text, or by way of the index (which is totally inadequate) is most noticeable and irritating in chapters which proceed by cataloguing rather than by synthesis. For example, the question of the biological importance of polymorphism in the major histocompatibility complex (MHC) comes up in the chapter on $\mathrm{T}$ and $B$ lymphocyte surface antigens in the sub-section on biochemistry of MHC antigens (p78), in the chapter on "Functional Properties of T Lymphocytes" during consideration of $\mathrm{H}-2$ restricted cytotoxic responses (pp 281-296) and in the chapter on genetic control of immune responses (p530 et seq.); and yet there is no cross-referencing of the discussions therein.

The organisation of the chapters under the chosen headings has led to some loss of clarity. This is particularly evident in the chapter entitled "Functional Properties of T Lymphocytes" subdivided into (A) "Regulatory $\mathrm{T}$ Lymphocytes" and (B) "Effector T lymphocytes". Such a classification, which quite arbitrarily considers helper and suppressor $\mathrm{T}$ lymphocytes under the first heading and mixed lymphocyte reaction proliferative $\mathrm{T}$ cells, cytotoxic $\mathrm{T}$ lymphocytes and $\mathrm{T}$ cells mediating delayed type hypersensitivity under the second, obscures the distinction between precursor $T$ cells for any particular function and effector cells which have differentiated as the result of exposure to antigen. The distinctive phenotype of the precursor cells for each function has been documented from many laboratories using anti-Ly antiserum, and implies a precommitment to function before encounter with antigen. This data on precommitment is contained in several of the references quoted in the bibliography but Dr Katz makes only brief mention of the notion, quoting just one of the references in a manner suggesting that it contains the only data available on the point (p265).

In a different context Ly phenotyping is mentioned under the heading of "Surface Phenotype Antigens" (chap ter VIII) and in the chapter on
Elizabeth Simpson

"Surface Markers of T Lymphocytes" (chapter II). Discussion of the data, however, is scattered in the text, and these are not used to integrate earlier, inevitably less well defined observations on the separation of functional subsets of $T$ cells. In some cases, as in the chapter on "Ontogeny of T Cells", they are not mentioned at all.

Chapters X, on "Regulatory Cellular Interactions", XII on "Genetic Control of Immune Responses" and XIII on "Mechanisms of Regulatory Cellular Interactions" obviously contain overlapping material. In some cases, the division chosen has fragmented and obscured the story; for example, the failure to follow through the discussion on $\mathrm{T}-\mathrm{B}$ cooperation in chapter $\mathrm{X}$ with the data on factors, which is presented in chapter XIII. The factor chapter (XIII) itself is incomplete in as much that the genetically restricted factor of Erb and Feldmann is mentioned not here, but in the previous chapter, to which there is no cross-reference on this point, and the suppressor factor of Kontiainen and Feldmann is not mentioned at all.

There are some misquotations of references, some by omission (that is, failing to take account of the whole of the contents of a paper, in some cases, stating that the author(s) had not looked at a particular point, when they had done so) and some by addition (that is, fanciful addition to the data); but this must be almost inevitable when so many references are considered, and does not occur often enough to mar the value of the text and its very extensive bibliography for serious readers. Informed immunologists will be able to seek out the discussion and references they want by assiduous detective work which would not have been necessary had the index been adequate and the cross-referencing in the text more thorough. For less informed readers-for example, graduate students--this valuable sourcebook and bibliography will not be so accessible.

Elizabeth Simpson works in the Division of Transplantation Biology at the Clinical Research, Centre, Harrow, UK. 